\title{
THE MEAN-FIELD LIMIT FOR SOLID PARTICLES IN A NAVIER-STOKES FLOW
}

\author{
LAURENT DESVILLETTES, FRANÇOIS GOLSE, AND VALERIA RICCI
}

\begin{abstract}
We propose a mathematical derivation of Brinkman's force for a cloud of particles immersed in an incompressible viscous fluid. Specifically, we consider the Stokes or steady Navier-Stokes equations in a bounded domain $\Omega \subset \mathbb{R}^{3}$ for the velocity field $u$ of an incompressible fluid with kinematic viscosity $\nu$ and density 1. Brinkman's force consists of a source term $6 \pi \nu j$ where $j$ is the current density of the particles, and of a friction term $6 \pi \nu \rho u$ where $\rho$ is the number density of particles. These additional terms in the motion equation for the fluid are obtained from the Stokes or steady Navier-Stokes equations set in $\Omega$ minus the disjoint union of $N$ balls of radius $\varepsilon=1 / N$ in the large $N$ limit with no-slip boundary condition. The number density $\rho$ and current density $j$ are obtained from the limiting phase space empirical measure $\frac{1}{N} \sum_{1<k<N} \delta_{x_{k}, v_{k}}$, where $x_{k}$ is the center of the $k$-th ball and $v_{k}$ its instantaneous velocity. This can be seen as a generalization of Allaire's result in [Arch. Rational Mech. Analysis 113 (1991) 209-259] who considered the case of periodically distributed $x_{k} \mathrm{~s}$ with $v_{k}=0$, and our proof is based on slightly simpler though similar homogenization arguments. Similar equations are used for describing the fluid phase in various models for sprays.
\end{abstract}

MSC: 35Q30, 35B27, 76M50

Key-words: Stokes equations, Navier-Stokes equations, Homogenization, Suspension flows

\section{INTRODUCTION}

The subject matter of this paper is the derivation of macroscopic models for the dynamics of large systems of solid particles or liquid droplets immersed in a viscous fluid (liquid or gas). Specifically, we are concerned with the collective effect of the friction force exerted on each particle as a result of the viscosity of the fluid together with a noslip condition at the surface of each particle. This type of fluid/solid interaction is relevant in several different physical contexts.

A first example is provided by the sedimentation of solid particles in a viscous incompressible fluid (say, a liquid), typically under the effect 
of gravity. There is a huge literature on this subject; we shall only mention a few, such as [3], [8], [5].

Another example is the case of sprays. Sprays are complex flows which consist of a dispersed phase immersed in some viscous fluid.

Sprays can be described (Cf. [14, 9]) by systems of coupled macroscopic equations (Eulerian-Eulerian modeling) or by the coupling of a macroscopic equation and a kinetic equation (Eulerian-Lagrangian modeling).

We do not claim that the results in this paper provide a complete derivation of any of these models for sprays, in particular because we do not analyze the coupling between the particle and the fluid dynamics. Also, our results apply to steady regimes only, for reasons that will be discussed below.

The present work is only aimed at providing a rigorous derivation of the Brinkman force created by a cloud of like spherical particles we recall that this force results from the collective effect of the drag exerted on the particles by the surrounding fluid. In models for sprays, this Brinkman force would typically be responsible for the coupling between the motion of the fluid and that of the dispersed phase.

Our approach of this problem is the homogenization method: we more or less follow earlier works such as [7] and [1] which only considered periodic distributions of particles. More precisely, the reference [7] established the friction term for the Laplace equation in a periodically perforated domain with homogeneous Dirichlet boundary condition. The case of the Stokes or Navier-Stokes equations was treated in [1] by similar arguments.

The discussion in the present paper differs from [1] in two ways. To begin with, only periodic distributions of particles all of which have the same velocity (which, by Galilean invariance can be taken as 0) are considered in [1]. In the present paper, we consider clouds of particles whose phase space empirical measure converges to some smooth phase space density. Thus, as long as this (mild) assumption is verified, the particles considered here can each have their own instantaneous velocity $^{1}$. Another difference with [1] lies in the method of proof, which may lead to simplifications here and there. The reference [1] closely followed the argument in [7] by truncating the velocity field in the vicinity of each particle, an operation that has the disadvantage of leading to velocity fields that fail to satisfy the incompressibility condition. In

\footnotetext{
${ }^{1}$ We mention also the paper [13], where an analogous problem is considered for the Navier-Stokes equations. This paper (as the references therein) does not unfortunately contain any detail about the convergence proof.
} 
the present work, the same goal is achieved by removing to the velocity field some carefully constructed solenoidal boundary layers so that the resulting vector field still satisfies the incompressibility condition. Hence the pressure can be integrated out, thereby leading to somewhat easier computations and avoiding painful estimates. Yet, our analysis borrows a lot from [7] and [1], especially in the construction of these boundary layers.

We found it convenient to describe the cloud of particles through its empirical measure instead of using (marginals of) its $N$-particle distribution function, as in [3], [8], [5], [6] — as a matter of fact, most of these references assume nearly factorized $N$-particle functions, so that both viewpoints are essentially equivalent.

\section{PRESentation of the MOdel AND MAin Results}

2.1. Formal derivation of the model. Consider a system of $N$ identical rigid spheres in a viscous incompressible fluid with kinematic viscosity $\nu$ and density $\rho_{f}$. For simplicity, we assume that the dynamics of the spheres is given, and we seek the collective effect on the fluid of the drag force on each sphere. We shall make the two following scaling assumptions:

a) the speed of the spheres is assumed to be small enough, so that the quasi-static approximation holds for the fluid motion, and

b) the collective effect of the drag forces exerted on each sphere is of the same order of magnitude as the external force field driving the fluid.

First, we outline the quasi-static approximation a). Our starting point is the set of Navier-Stokes equations

$$
\begin{aligned}
\partial_{t} u+u \cdot \nabla_{x} u+\nabla_{x} p & =\nu \triangle_{x} u+f, \quad \nabla_{x} \cdot u=0, \\
\left.u(t, \cdot)\right|_{\partial B\left(x_{k}(t), r\right)} & =\dot{x}_{k}(t),
\end{aligned}
$$

where $u \equiv u(t, x) \in \mathbb{R}^{3}$ and $p \equiv p(t, x)$ are respectively the velocity and pressure field in the fluid, while $r$ is the radius of the rigid balls immersed in the fluid and $x_{k}(t)$ is the position at time $t$ of the center of the $k$-th ball $B_{x_{k}(t), r}$. The density of external force per unit of mass in the fluid is $f \equiv f(t, x) \in \mathbb{R}^{3}$.

Notice that, in this model, the effect of solid rotation for each particle is neglected - together with the amount of torque particles subject to such solid rotations would exert on the fluid.

Assume that the motion of the spheres occurs at a time scale that is long compared to the typical time scale of the external force field $f$. In other words, we postulate the existence of a small parameter $\tau \ll 1$ 
such that

$$
x_{k}(t)=X_{k}(\tau t)
$$

The quasi-static approximation is obtained as follows: defining the slow time variable $T=\tau t$ and $u(t, x)=\tau U(\tau t, x)$, the left-hand side of the Navier-Stokes equation is rescaled as

$$
\partial_{t} u+u \cdot \nabla_{x} u-\nu \triangle_{x} u=\tau^{2}\left(\partial_{T} U+U \cdot \nabla_{x} U\right)-\tau \nu \triangle_{x} U
$$

Defining

$$
\tau F(T, x)=f\left(\frac{T}{\tau}, x\right), \tau P(T, x)=p\left(\frac{T}{\tau}, x\right), \quad \text { and } V_{k}=\frac{d X_{k}}{d T}
$$

we recast the Navier-Stokes problem above

$$
\begin{aligned}
\tau^{2}\left(\partial_{T} U+U \cdot \nabla_{x} U\right)+\tau \nabla_{x} P & =\tau\left(\nu \triangle_{x} U+F\right), \quad \nabla_{x} \cdot U=0 \\
\left.U(T, \cdot)\right|_{\partial B_{X_{k}(T), r}} & =V_{k}(T) .
\end{aligned}
$$

Neglecting all terms of order $O\left(\tau^{2}\right)$ in $(2)$, we arrive at the quasi-static Stokes problem

$$
\begin{aligned}
-\nu \triangle_{x} U+\nabla_{x} P & =F, \quad \nabla_{x} \cdot U=0, \\
\left.U(T, \cdot)\right|_{\partial B_{X_{k}(T), r}} & =V_{k}(T) .
\end{aligned}
$$

Notice that, in the Stokes problem above, $T$ is only a parameter, so that $X_{k}(T)$ and $V_{k}(T)$ can be regarded as independent. In other words, in the Stokes problem considered below, it will be legitimate, under the quasi-static approximation, to consider $X_{k}$ as a constant and yet to allow $V_{k} \neq 0$.

This accounts for item a) above in the derivation of our model; let us now discuss item b), namely the collective effect of the drag force exerted on the spheres.

We recall that the drag force exerted on a single sphere of radius $r$ immersed in a Stokes fluid with kinematic viscosity $\nu$, density $\rho_{f}$ is

$$
6 \pi \rho_{f} \nu r V
$$

where $V$ is the relative velocity of the sphere - relatively to the speed of the fluid at infinity: see [12] $\S 20$.

Hence the collective force field exerted on the fluid by a system of $N$ identical such spheres with prescribed dynamics is of the order of

$$
6 \pi \rho_{f} \nu N r\langle V\rangle
$$

where $\langle V\rangle$ is the average relative velocity of the spheres.

In the sequel, we assume that the parameters $\nu$ and $\rho_{f}$ are of order $O(1)$, as well as $\langle V\rangle$, but we are interested in situations where $r \ll 1$ (small spheres) and $N \gg 1$ (large number of spheres). In order for the collective effect of the immersed spheres to be of the same order 
as that of the driving external force field, we postulate (without loss of generality) that

$$
N r \simeq \text { Const. }>0 \text {. }
$$

This scaling assumption leads to the mean field approximation listed above as b).

2.2. The quasi-static, mean field limit. Henceforth we use the sphere radius as the small parameter governing all limits of interest here, and denote it by $\varepsilon>0$ instead of $r$. Thus we assume that $N \rightarrow \infty, \varepsilon \rightarrow 0$ and

$$
N \varepsilon=1
$$

We further assume that the fluid and the particles considered here are enclosed in a domain $\Omega$ and denote the volume that is left free for fluid motion by

$$
\Omega_{\varepsilon}=\Omega \backslash \bigcup_{k=1}^{N} B_{x_{k}, \varepsilon} .
$$

In this setting, the Stokes problem for the velocity field $u_{\varepsilon}$ and the pressure field $p_{\varepsilon}$ reads

$$
\left\{\begin{aligned}
-\triangle u_{\varepsilon}+\nabla p_{\varepsilon} & =g, \\
\nabla \cdot u_{\varepsilon} & =0,
\end{aligned} \quad \text { on } \Omega_{\varepsilon} .\right.
$$

Here, the source term $g$ is the ratio of density of external force per unit of mass to the kinematic viscosity. This system is supplemented with a no-slip boundary conditions for $u$ on the boundary of $\Omega_{\varepsilon}$ :

$$
\left\{\begin{aligned}
\left.u\right|_{\partial B_{x_{k}, \varepsilon}} & =v_{k}, \quad \text { for } \quad k=1, . ., N, \\
\left.u\right|_{\partial \Omega} & =0,
\end{aligned}\right.
$$

where $v_{k}$ is the instantaneous velocity of the (center of mass of the) $k$-th sphere.

Denote by

$$
F_{N}(x, v)=\frac{1}{N} \sum_{k=1}^{N} \delta_{x_{k}, v_{k}}(x, v)
$$

the phase space empirical measure of the system of $N$ spheres and by

$$
\rho_{N}(x)=\int_{\mathbb{R}^{3}} F_{N}(x, v) d v ; \quad j_{N}(x)=\int_{\mathbb{R}^{3}} F_{N}(x, v) v d v
$$

its two first moments. 
It will be convenient to consider the natural extension of $u_{\varepsilon}$ to $\Omega$ defined by

$$
\bar{u}_{\varepsilon}(x)= \begin{cases}u_{\varepsilon}(x) & \text { if } \quad x \in \Omega_{\varepsilon}, \\ v_{k} & \text { if } \quad x \in B_{x_{k}, \varepsilon}, \quad k=1, \ldots, N .\end{cases}
$$

As recalled above, the Stokes' computation of the friction exerted on an immersed sphere by the surrounding viscous incompressible fluid involves the relative velocity of the sphere to the speed of the fluid at infinity. In order to extend Stokes' analysis to the mean field situation considered here, we need to assume that the distance between the immersed particles is large enough compared to their size. Specifically, we assume that

$$
\inf _{1 \leq k \neq l \leq N}\left|x_{k}-x_{l}\right|>2 r_{\varepsilon} \text { where } r_{\varepsilon}:=\varepsilon^{1 / 3} .
$$

This assumption on the distance between particles is consistent with the critical scale for the total number of particles discussed in [10]. The assumption (11) allows considering each particle subject to a drag force given by Stokes' formula independently of other particles. Obviously, we do not know whether (11) is preserved under particle motion, and this is why only steady situations are considered here.

Likewise, we assume for simplicity that the fluid and the particles occupy a smooth bounded domain $\Omega \subset \mathbb{R}^{3}$, and that there is no direct interaction between the boundary of $\Omega$ and any of the immersed particles:

$$
\inf _{1 \leq k \leq N} \operatorname{dist}\left(x_{k}, \partial \Omega\right)>r_{\varepsilon} .
$$

Theorem 1. Let $\Omega \subset \mathbb{R}^{3}$ be a smooth bounded domain, and consider a system of $N$ balls $B_{x_{k}, \varepsilon}$ for $k=1, \ldots, N$ and $\varepsilon=1 / N$ included in $\Omega$ and satisfying conditions (11)-(12). Assume that the empirical measure $F_{N}$ has uniformly bounded kinetic energy

$$
\sup _{N \geq 1} \iint_{\Omega \times \mathbb{R}^{3}} \frac{1}{2}|v|^{2} F_{N}(x, v) d x d v<\infty
$$

while the macroscopic density and the current converge weakly in the sense of measures

$$
\rho_{N} \rightarrow \rho, \quad j_{N} \rightarrow j \text { as } N \rightarrow \infty
$$

with $\rho$ and $j$ continuous on $\bar{\Omega}$.

For each $g \in\left(L^{2}(\Omega)\right)^{3}$, let $u_{\varepsilon}$ be the unique weak solution in $\left(H^{1}\left(\Omega_{\varepsilon}\right)\right)^{3}$ of (6), (7), and define $\bar{u}_{\varepsilon}$ as in (10). Then, $\bar{u}_{\varepsilon}$ converges in $\left(L^{2}(\Omega)\right)^{3}$ 
to the solution $U$ of

$$
\left\{\begin{aligned}
-\Delta U+\nabla \Pi & =g+6 \pi(j-\rho U), \\
\nabla \cdot U & =0 \\
\left.U\right|_{\partial \Omega} & =0
\end{aligned}\right.
$$

As a matter of fact, the same techniques as in the proof of Theorem 1 allow considering the steady Navier-Stokes, instead of Stokes equations. The starting point in this case is

$$
\left\{\begin{aligned}
u_{\varepsilon} \cdot \nabla u_{\varepsilon}-\nu \triangle u_{\varepsilon}+\nabla p_{\varepsilon} & =g, \\
\nabla \cdot u_{\varepsilon} & =0,
\end{aligned} \quad \text { on } \Omega_{\varepsilon} .\right.
$$

In writing the system above, we have retained the kinematic viscosity $\nu$ instead of absorbing it in the source term as in the linear, Stokes case. Hence, unlike in (6), $g$ is the density of external force per unit of mass (instead of its ratio to the kinematic viscosity).

The limiting equations in this case are

$$
\left\{\begin{aligned}
U \cdot \nabla U-\nu \triangle U+\nabla \Pi & =g+6 \pi \nu(j-\rho U), \\
\nabla \cdot U & =0, \\
\left.U\right|_{\partial \Omega} & =0
\end{aligned}\right.
$$

Let us briefly discuss the uniqueness problem for (15). By a standard energy argument, one finds that, if $U_{1}$ and $U_{2}$ are weak solutions of (15), they must satisfy

$$
\begin{aligned}
6 \pi \nu \int_{\Omega} \rho\left|U_{1}-U_{2}\right|^{2} d x & +\nu\left\|\nabla\left(U_{1}-U_{2}\right)\right\|_{L^{2}(\Omega)}^{2} \\
& \leq\left\|\nabla U_{1}\right\|_{L^{2}(\Omega)}\left\|U_{1}-U_{2}\right\|_{L^{4}(\Omega)}^{2} \\
& +\left\|U_{2}\right\|_{L^{4}(\Omega)}\left\|\nabla\left(U_{1}-U_{2}\right)\right\|_{L^{2}(\Omega)}\left\|U_{1}-U_{2}\right\|_{L^{4}(\Omega)}
\end{aligned}
$$

We first recall (see [11] p. 9) that

$$
\left\|U_{\varepsilon}\right\|_{L^{4}(\Omega)}^{4} \leq 4\left\|U_{\varepsilon}\right\|_{L^{2}(\Omega)}\left\|\nabla U_{\varepsilon}\right\|_{L^{2}(\Omega)}^{3} ;
$$

together with the Poincaré inequality, this entails

$$
\left\|U_{\varepsilon}\right\|_{L^{4}(\Omega)}^{4} \leq 4 C_{P}\left\|\nabla U_{\varepsilon}\right\|_{L^{2}(\Omega)}^{4}
$$

where $C_{P}$ denotes the Poincaré constant in the domain $\Omega$. Hence

$$
\begin{aligned}
& \nu\left\|\nabla\left(U_{1}-U_{2}\right)\right\|_{L^{2}(\Omega)}^{2} \\
& \quad \leq 2 C_{P}^{1 / 2}\left(\left\|\nabla U_{1}\right\|_{L^{2}(\Omega)}+\left\|\nabla U_{2}\right\|_{L^{2}(\Omega)}\right)\left\|\nabla\left(U_{1}-U_{2}\right)\right\|_{L^{2}(\Omega)}^{2} .
\end{aligned}
$$

Therefore, uniqueness holds for (15) if

$$
\nu \geq 2 C_{P}^{1 / 2}\left(\left\|\nabla U_{1}\right\|_{L^{2}(\Omega)}+\left\|\nabla U_{2}\right\|_{L^{2}(\Omega)}\right) .
$$


But the usual energy estimates for either of the weak solutions $U_{1}$ and $U_{2}$ shows that

$$
\nu\left\|\nabla U_{j}\right\|_{L^{2}(\Omega)} \leq C_{P}\left(\|g\|_{L^{2}(\Omega)}+6 \pi \nu\|j\|_{L^{2}(\Omega)}\right) .
$$

Finally, uniqueness holds for (15) if

$$
\nu^{2} \geq 4 C_{P}^{3 / 2}\left(\|g\|_{L^{2}(\Omega)}+6 \pi \nu\|j\|_{L^{2}(\Omega)}\right)
$$

i.e. for $\nu>\nu_{0} \equiv \nu_{0}\left(\|g\|_{L^{2}(\Omega)},\|j\|_{L^{2}(\Omega)}, C_{P}\right)$.

Theorem 2. Under the same assumptions as in Theorem 1 and for each $\nu>\nu_{0}\left(\|g\|_{L^{2}(\Omega)},\|j\|_{L^{2}(\Omega)}, C_{P}\right)$, consider, for each $g \in L^{2}(\Omega)$ and each $\varepsilon=1 / N$, a solution $u_{\varepsilon}$ of the steady Navier-Stokes equations (14) with the no-slip boundary condition (7). Defining its natural extension to $\Omega$ to be $\bar{u}_{\varepsilon}$ as in (10), one has $\bar{u}_{\varepsilon} \rightarrow u$ in $L^{2}(\Omega)$ as $\varepsilon=1 / N \rightarrow 0$, where $u$ is the unique weak solution of (15).

\section{METHOD OF PROOF}

In this section, we present the strategy for the proofs of Theorems 1 and 2 .

3.1. Introducing correctors. We recall that the weak formulation of the Stokes problem (6)-(7) is

$$
\int_{\Omega_{\varepsilon}} \nabla u_{\varepsilon} \cdot \nabla W d x=\int_{\Omega_{\varepsilon}} g \cdot W d x
$$

while the weak formulation of the Navier-Stokes problem (14), (7) is

$$
\nu \int_{\Omega_{\varepsilon}} \nabla u_{\varepsilon} \cdot \nabla W d x=\int_{\Omega_{\varepsilon}} u_{\varepsilon} \otimes u_{\varepsilon}: \nabla W d x+\int_{\Omega_{\varepsilon}} g \cdot W d x,
$$

for each test solenoidal vector field $W \in\left(H_{0}^{1}\left(\Omega_{\varepsilon}\right)\right)^{3}$, i.e. such that $\nabla \cdot W=0$.

For each $w \in(\mathcal{D}(\Omega))^{3}$ such that $\nabla \cdot w=0$, we choose test vector fields of the form

$$
W_{\varepsilon}=w-\mathcal{B}_{\varepsilon}[w]
$$

where $\mathcal{B}_{\varepsilon}[w] \in\left(H_{0}^{1}(\Omega)\right)^{3}$ satisfies

$$
\nabla \cdot \mathcal{B}_{\varepsilon}[w]=0 \text { in } \Omega \text { and }\left.\mathcal{B}_{\varepsilon}[w]\right|_{\bar{B}_{x_{k}, \varepsilon}}=\left.w\right|_{\bar{B}_{x_{k}, \varepsilon}} .
$$

Similarly, we approximate the solution by

$$
U_{\varepsilon}=\bar{u}_{\varepsilon}-\mathcal{A}_{\varepsilon}
$$

where $\mathcal{A}_{\varepsilon} \in\left(H_{0}^{1}(\Omega)\right)^{3}$ satisfies

$$
\nabla \cdot \mathcal{A}_{\varepsilon}=0 \text { in } \Omega \text { and }\left.\mathcal{A}_{\varepsilon}\right|_{\bar{B}_{x_{k}, \varepsilon}}=v_{k} .
$$


Explicit formulas for the fields $\mathcal{A}_{\varepsilon}$ and $\mathcal{B}_{\varepsilon}$ will be given at the end of the present section. Notice that, by construction,

$$
\left.U_{\varepsilon}\right|_{B_{x_{k}, \varepsilon}}=\left.W_{\varepsilon}\right|_{B_{x_{k}, \varepsilon}}=0, \quad \text { for all } k=1, . ., N \text {. }
$$

In addition, the correctors $\mathcal{A}_{\varepsilon}$ and $\mathcal{B}_{\varepsilon}$ are chosen so that

$$
\begin{aligned}
\mathcal{B}_{\varepsilon}[w] & \rightarrow 0 \text { in }\left(H_{0}^{1}(\Omega)\right)^{3}, \\
\mathcal{A}_{\varepsilon} \rightarrow 0 & \text { in }\left(H_{0}^{1}(\Omega)\right)^{3} .
\end{aligned}
$$

Condition (19) implies that

$$
W_{\varepsilon} \rightarrow w \text { in }\left(H_{0}^{1}(\Omega)\right)^{3} \text { and } \nabla W_{\varepsilon} \rightarrow \nabla w \text { in }\left(L^{2}(\Omega)\right)^{9} .
$$

Moreover, (19) and (20) imply that

$$
\begin{aligned}
\mathcal{B}_{\varepsilon}[w] & \rightarrow 0 \text { in }\left(L^{p}(\Omega)\right)^{3}, \\
\mathcal{A}_{\varepsilon} & \rightarrow 0 \text { in }\left(L^{p}(\Omega)\right)^{3},
\end{aligned}
$$

for each $p \in[1,6)$, by the Rellich-Kondrachov compact embedding theorem, so that

$$
W_{\varepsilon} \rightarrow w \text { in }\left(L^{p}(\Omega)\right)^{3} \text { for each } p \in[1,6) .
$$

Condition (20) implies that $U_{\varepsilon}$ and $\bar{u}_{\varepsilon}$ behave similarly as $\varepsilon \rightarrow 0$. In the next subsection, we study the asymptotic behavior of $U_{\varepsilon}$, which is somewhat simpler to analyze. As we shall see, condition (20) implies that

$$
U_{\varepsilon} \rightarrow U \text { in }\left(H_{0}^{1}(\Omega)\right)^{3} \text { as } \varepsilon \rightarrow 0
$$

for both problems (6) and (14) with the boundary condition (7). Hence

$$
U_{\varepsilon} \rightarrow U \text { in }\left(L^{p}(\Omega)\right)^{3} \text { for } 1 \leq p<6
$$

as $\varepsilon \rightarrow 0$.

3.2. Weak convergence of $U_{\varepsilon}$. Here we show that (some subsequence of) $U_{\varepsilon}$ converges weakly in $\left(H^{1}(\Omega)\right)^{3}$ (assuming (19) and (20)), for both problems (6) and (14) with boundary condition (7).

Indeed, for each $k=1, \ldots, N$, one has $\left.U_{\varepsilon}\right|_{B_{x_{k}, \varepsilon}}=0$, so that the weak formulation of the Stokes problem becomes :

$$
\begin{aligned}
\left\|\nabla U_{\varepsilon}\right\|_{L^{2}(\Omega)}^{2} & =\int_{\Omega} \nabla \bar{u}_{\varepsilon}: \nabla U_{\varepsilon} d x-\int_{\Omega} \nabla \mathcal{A}_{\varepsilon}: \nabla U_{\varepsilon} d x \\
& =\int_{\Omega} g \cdot U_{\varepsilon} d x-\int_{\Omega} \nabla \mathcal{A}_{\varepsilon}: \nabla U_{\varepsilon} d x \\
& \leq\|g\|_{L^{2}(\Omega)}\left\|U_{\varepsilon}\right\|_{L^{2}(\Omega)}+\left\|\nabla \mathcal{A}_{\varepsilon}\right\|_{L^{2}(\Omega)}\left\|\nabla U_{\varepsilon}\right\|_{L^{2}(\Omega)}
\end{aligned}
$$

By the Poincaré inequality and (20), which entails a uniform bound of the form $\left\|\nabla \mathcal{A}_{\varepsilon}\right\|_{L^{2}(\Omega)}<C$, we conclude that $\left\|\nabla U_{\varepsilon}\right\|_{L^{2}(\Omega)}$ is bounded. Hence there is a subsequence such that $U_{\varepsilon} \rightarrow U$ in $\left(H^{1}(\Omega)\right)^{3}$. 
For the Navier-Stokes problem we have similarly:

$$
\begin{aligned}
& \nu\left\|\nabla U_{\varepsilon}\right\|_{L^{2}(\Omega)}^{2}=\nu \int_{\Omega} \nabla \bar{u}_{\varepsilon}: \nabla U_{\varepsilon} d x-\nu \int_{\Omega} \nabla \mathcal{A}_{\varepsilon}: \nabla U_{\varepsilon} d x \\
& =\int_{\Omega} g \cdot U_{\varepsilon} d x-\int_{\Omega_{\varepsilon}}\left(\bar{u}_{\varepsilon} \cdot \nabla \bar{u}_{\varepsilon}\right) \cdot U_{\varepsilon} d x-\nu \int_{\Omega} \nabla \mathcal{A}_{\varepsilon}: \nabla U_{\varepsilon} d x \\
& =\int_{\Omega} g \cdot U_{\varepsilon} d x+\int_{\Omega_{\varepsilon}} U_{\varepsilon} \otimes U_{\varepsilon}: \nabla U_{\varepsilon} d x-\nu \int_{\Omega} \nabla \mathcal{A}_{\varepsilon}: \nabla U_{\varepsilon} d x \\
& +\int_{\Omega_{\varepsilon}}\left(\mathcal{A}_{\varepsilon} \otimes U_{\varepsilon}+U_{\varepsilon} \otimes \mathcal{A}_{\varepsilon}+\mathcal{A}_{\varepsilon} \otimes \mathcal{A}_{\varepsilon}\right): \nabla U_{\varepsilon} d x .
\end{aligned}
$$

Observe that

$$
\begin{aligned}
\int_{\Omega_{\varepsilon}} U_{\varepsilon} \otimes U_{\varepsilon}: \nabla U_{\varepsilon} d x & =\int_{\Omega_{\varepsilon}} U_{\varepsilon} \cdot\left(\left(U_{\varepsilon} \cdot \nabla\right) U_{\varepsilon}\right) d x \\
& =\frac{1}{2} \int_{\Omega_{\varepsilon}} \nabla\left(U_{\varepsilon}\left|U_{\varepsilon}\right|^{2}\right) d x=0
\end{aligned}
$$

by Green's formula, since $\left.U_{\varepsilon}\right|_{\partial \Omega_{\varepsilon}}=0$. Hence

$$
\begin{aligned}
\nu \| \nabla & U_{\varepsilon}\left\|_{L^{2}(\Omega)}^{2} \leq\right\| g\left\|_{L^{2}(\Omega)}\right\| U_{\varepsilon}\left\|_{L^{2}(\Omega)}+\nu\right\| \nabla \mathcal{A}_{\varepsilon}\left\|_{L^{2}(\Omega)}\right\| \nabla U_{\varepsilon} \|_{L^{2}(\Omega)} \\
& +\left(\left\|\mathcal{A}_{\varepsilon} \otimes U_{\varepsilon}\right\|_{L^{2}(\Omega)}+\left\|U_{\varepsilon} \otimes \mathcal{A}_{\varepsilon}\right\|_{L^{2}(\Omega)}+\left\|\mathcal{A}_{\varepsilon}^{\otimes 2}\right\|_{L^{2}(\Omega)}\right)\left\|\nabla U_{\varepsilon}\right\|_{L^{2}(\Omega)} \\
& \leq\|g\|_{L^{2}(\Omega)}\left\|U_{\varepsilon}\right\|_{L^{2}(\Omega)} \\
& +\left(\nu\left\|\nabla \mathcal{A}_{\varepsilon}\right\|_{L^{2}(\Omega)}+2\left\|\mathcal{A}_{\varepsilon}\right\|_{L^{4}(\Omega)}\left\|U_{\varepsilon}\right\|_{L^{4}(\Omega)}+\left\|\mathcal{A}_{\varepsilon}\right\|_{L^{4}(\Omega)}^{2}\right)\left\|\nabla U_{\varepsilon}\right\|_{L^{2}(\Omega)}
\end{aligned}
$$

Applying inequality (16) shows that

$$
\begin{aligned}
\left(\nu-2 \sqrt{2} C_{P}^{1 / 4}\left\|\mathcal{A}_{\varepsilon}\right\|_{L^{4}(\Omega)}\right) & \left\|\nabla U_{\varepsilon}\right\|_{L^{2}(\Omega)} \\
\leq & \left(C_{P}\|g\|_{L^{2}(\Omega)}+\nu\left\|\nabla \mathcal{A}_{\varepsilon}\right\|_{L^{2}(\Omega)}+\left\|\mathcal{A}_{\varepsilon}\right\|_{L^{4}(\Omega)}^{2}\right) .
\end{aligned}
$$

Recall that $\left\|\mathcal{A}_{\varepsilon}\right\|_{L^{4}(\Omega)} \rightarrow 0$ as $\varepsilon \rightarrow 0$ by $(21)$, while $\left\|\nabla \mathcal{A}_{\varepsilon}\right\|_{L^{2}(\Omega)} \leq C$ by (20). Hence the estimate above entails the bound

$$
\left\|\nabla U_{\varepsilon}\right\|_{L^{2}(\Omega)} \leq C \text {. }
$$

3.3. Weak formulations on the whole domain. Next we recast the weak formulations (17) and (18) in terms of $U_{\varepsilon}$ : as we shall see, this is somewhat more convenient, at least in taking the mean field limit.

We first discuss the Stokes problem (17). Observe that

$$
\int_{\Omega} \nabla \bar{u}_{\varepsilon}: \nabla W_{\varepsilon} d x=\int_{\Omega_{\varepsilon}} \nabla u_{\varepsilon}: \nabla W_{\varepsilon} d x=\int_{\Omega_{\varepsilon}} g \cdot W_{\varepsilon} d x .
$$


Expressing $\bar{u}_{\varepsilon}$ in terms of $U_{\varepsilon}$, one arrives at

$$
\int_{\Omega} \nabla U_{\varepsilon}: \nabla W_{\varepsilon} d x+\int_{\Omega} \nabla \mathcal{A}_{\varepsilon}: \nabla W_{\varepsilon} d x=\int_{\Omega} g \cdot W_{\varepsilon} d x
$$

which, after replacing $W_{\varepsilon}$ with its expression in terms of $w$, leads to

$$
\int_{\Omega} \nabla U_{\varepsilon}: \nabla w d x-\int_{\Omega} \nabla U_{\varepsilon}: \nabla \mathcal{B}_{\varepsilon} d x+\int_{\Omega} \nabla \mathcal{A}_{\varepsilon}: \nabla W_{\varepsilon} d x=\int_{\Omega} g \cdot W_{\varepsilon} d x .
$$

Transforming the second integral on the right hand side by Green's formula, one eventually obtains

$$
\int_{\Omega} \nabla U_{\varepsilon}: \nabla w d x+\int_{\Omega} U_{\varepsilon} \cdot \triangle \mathcal{B}_{\varepsilon} d x-\int_{\Omega} \triangle \mathcal{A}_{\varepsilon} \cdot W_{\varepsilon} d x=\int_{\Omega} g \cdot W_{\varepsilon} d x .
$$

Under assumptions (19)-(20)

$$
\begin{aligned}
\int_{\Omega} \nabla U_{\varepsilon}: \nabla w d x & \rightarrow \int_{\Omega} \nabla U: \nabla w d x \\
\int_{\Omega} g \cdot W_{\varepsilon} d x & \rightarrow \int_{\Omega} g \cdot w d x
\end{aligned}
$$

as $\varepsilon \rightarrow 0$. Thus we are left with computing the limit of

$$
-\int_{\Omega} \nabla U_{\varepsilon}: \nabla \mathcal{B}_{\varepsilon} d x+\int_{\Omega} \nabla \mathcal{A}_{\varepsilon}: \nabla W_{\varepsilon} d x
$$

or, equivalently, of

$$
\int_{\Omega} U_{\varepsilon} \cdot \triangle \mathcal{B}_{\varepsilon} d x-\int_{\Omega} \triangle \mathcal{A}_{\varepsilon} \cdot W_{\varepsilon} d x .
$$

For the Navier-Stokes problem (18), we follow the same arguments. First

$$
\begin{aligned}
& \nu \int_{\Omega_{\varepsilon}} \nabla u_{\varepsilon} \cdot \nabla W_{\varepsilon} d x-\int_{\Omega_{\varepsilon}} u_{\varepsilon} \otimes u_{\varepsilon}: \nabla W_{\varepsilon} d x \\
& =\nu \int_{\Omega} \nabla \bar{u}_{\varepsilon} \cdot \nabla W_{\varepsilon} d x-\int_{\Omega} \bar{u}_{\varepsilon} \otimes \bar{u}_{\varepsilon}: \nabla W_{\varepsilon} d x=\int_{\Omega} g \cdot W_{\varepsilon} d x
\end{aligned}
$$

since $v_{k}, k=1, \ldots, N$, are constants and $\left.W_{\varepsilon}\right|_{B_{x_{k}, \varepsilon}}=0$. Making the substitution $\bar{u}_{\varepsilon}=U_{\varepsilon}+\mathcal{A}_{\varepsilon}$ in (25), one gets

$$
\begin{aligned}
& \nu \int_{\Omega} \nabla U_{\varepsilon}: \nabla W_{\varepsilon} d x+\nu \int_{\Omega} \nabla \mathcal{A}_{\varepsilon}: \nabla W_{\varepsilon} d x=\int_{\Omega} U_{\varepsilon} \otimes U_{\varepsilon}: \nabla W_{\varepsilon} d x \\
& \quad+\int_{\Omega} g \cdot W_{\varepsilon} d x+\int_{\Omega}\left(\mathcal{A}_{\varepsilon} \otimes U_{\varepsilon}+U_{\varepsilon} \otimes \mathcal{A}_{\varepsilon}+\mathcal{A}_{\varepsilon} \otimes \mathcal{A}_{\varepsilon}\right): \nabla W_{\varepsilon} d x
\end{aligned}
$$


and, inserting $W_{\varepsilon}=w-\mathcal{B}_{\varepsilon}$ in the equality above, one eventually arrives at

$$
\begin{array}{r}
\nu \int_{\Omega} \nabla U_{\varepsilon}: \nabla w d x-\nu \int_{\Omega} \nabla U_{\varepsilon}: \nabla \mathcal{B}_{\varepsilon} d x+\nu \int_{\Omega} \nabla \mathcal{A}_{\varepsilon}: \nabla W_{\varepsilon} d x \\
=\int_{\Omega} U_{\varepsilon} \otimes U_{\varepsilon}: \nabla W_{\varepsilon} d x+\int_{\Omega} g \cdot W_{\varepsilon} d x \\
+\int_{\Omega}\left(\mathcal{A}_{\varepsilon} \otimes U_{\varepsilon}+U_{\varepsilon} \otimes \mathcal{A}_{\varepsilon}+\mathcal{A}_{\varepsilon} \otimes \mathcal{A}_{\varepsilon}\right): \nabla W_{\varepsilon} d x .
\end{array}
$$

Next we pass to the limit as $\varepsilon \rightarrow 0$; assumptions (19)-(20) imply that $\mathcal{A}_{\varepsilon}$ and $U_{\varepsilon}$ converge strongly in $L^{4}(\Omega)$, so that

$$
\begin{aligned}
\int_{\Omega}\left(\mathcal{A}_{\varepsilon} \otimes U_{\varepsilon}+U_{\varepsilon} \otimes \mathcal{A}_{\varepsilon}+\mathcal{A}_{\varepsilon} \otimes \mathcal{A}_{\varepsilon}\right): \nabla W_{\varepsilon} d x & \rightarrow 0 \\
\int_{\Omega} U_{\varepsilon} \otimes U_{\varepsilon}: \nabla W_{\varepsilon} d x & \rightarrow \int_{\Omega} U \otimes U: \nabla w d x .
\end{aligned}
$$

Moreover

$$
\begin{aligned}
\int_{\Omega} g \cdot W_{\varepsilon} d x & \rightarrow \int_{\Omega} g \cdot w d x, \\
\nu \int_{\Omega} \nabla U_{\varepsilon}: \nabla w d x & \rightarrow \nu \int_{\Omega} \nabla U: \nabla w d x,
\end{aligned}
$$

so that we are left with the task of computing the limit as $\varepsilon \rightarrow 0$ of

$$
-\nu \int_{\Omega} \nabla U_{\varepsilon}: \nabla \mathcal{B}_{\varepsilon} d x+\nu \int_{\Omega} \nabla \mathcal{A}_{\varepsilon}: \nabla W_{\varepsilon} d x .
$$

At this point, we need to specify how the correctors $\mathcal{A}_{\varepsilon}$ and $\mathcal{B}_{\varepsilon}$ are constructed.

3.4. Defining the correctors. Given any smooth function $w$ on $B_{0, s}$ and $r>s$, we designate by $\Psi_{s, r}[w]$ the solution of the following Stokes problem :

$$
\left\{\begin{array}{l}
\triangle \Psi_{s, r}[w]=\nabla \Pi_{s, r}[w], \quad x \in B_{0, r} \backslash B_{0, s} \\
\nabla \cdot \Psi_{s, r}[w]=0 \\
\left.\Psi_{s, r}[w]\right|_{B_{0, s}}=w \\
\left.\Psi_{s, r}[w]\right|_{B_{0, r}^{c}} ^{c}=0
\end{array}\right.
$$

When $s=\varepsilon, r=r_{\varepsilon}=\varepsilon^{1 / 3}$, we define

$$
\psi_{\varepsilon}[w]=\Psi_{\varepsilon, \varepsilon^{1 / 3}}[w] .
$$

We denote $\left.\pi_{\varepsilon}[w]=\Pi_{\varepsilon, \varepsilon^{1 / 3}}[w]\right)$, the pressure field associated to $\psi_{\varepsilon}[w]$. 
With the function $\psi_{\varepsilon}[w]$, we define the corrector $\mathcal{B}_{\varepsilon}$ as follows:

$$
\mathcal{B}_{\varepsilon}=\sum_{k=1}^{N} \psi_{\varepsilon}\left[w\left(\cdot+x_{k}\right)\right]\left(x-x_{k}\right) \text {. }
$$

Whenever $w$ is a constant, i.e. $w(x)=v$, we use the notation

$$
\Phi_{s, r}[v]=\Psi_{s, r}[w] \text { and likewise } \phi_{\varepsilon}[v]=\psi_{\varepsilon}[w] \text {. }
$$

With the function $\phi_{\varepsilon}[v]$, we define the corrector $\mathcal{A}_{\varepsilon}$ in the following manner:

$$
\mathcal{A}_{\varepsilon}=\sum_{k=1}^{N} \phi_{\varepsilon}\left[v_{k}\right]\left(x-x_{k}\right) .
$$

The vector fields $\mathcal{A}_{\varepsilon}$ and $\mathcal{B}_{\varepsilon}$ so defined are obviously solenoidal elements of $\left(H_{0}^{1}(\Omega)\right)^{3}$ that verify the conditions

$$
\left.\mathcal{A}_{\varepsilon}\right|_{\bar{B}_{x_{k}, \varepsilon}}=v_{k} \text { and }\left.\mathcal{B}_{\varepsilon}[w]\right|_{\bar{B}_{x_{k}, \varepsilon}}=\left.w\right|_{\bar{B}_{x_{k}, \varepsilon}} .
$$

In section 5 , we shall prove that $\mathcal{A}_{\varepsilon}$ and $\mathcal{B}_{\varepsilon}$ verify assumptions (20)-(19).

\section{EXPLICIT FORMULAS FOR THE CORRECTORS}

The Stokes equations in an annulus can be solved explicitly; in this section, we use these explicit formula to express the correctors $\mathcal{A}_{\varepsilon}$ and $\mathcal{B}_{\varepsilon}$, and to estimate the quantity

$$
\int_{\Omega} \nabla U_{\varepsilon}: \nabla \mathcal{B}_{\varepsilon} d x-\int_{\Omega} \nabla \mathcal{A}_{\varepsilon}: \nabla W_{\varepsilon} d x
$$

Occasionally, we will refer to the appendix (section 6.2) where a few standard computations are summarized.

We start with a formula for $\Phi_{1, R}[v]$ - and hence for $\phi_{\varepsilon}[v]$. For each $x \in \mathbb{R}^{3}$, denote $r=|x|, \omega=\frac{x}{|x|}$. Moreover, we denote $P_{\omega} a=(\omega \cdot a) \omega$ is the orthogonal projection on the line $\mathbb{R} \omega$.

Whenever $1 \leq r \leq R$,

$$
\begin{aligned}
\Phi_{1, R}[v](x)= & -\left[4 \alpha(R) r^{2}+2 \beta(R)+\frac{\gamma(R)}{r}-\frac{\delta(R)}{r^{3}}\right]\left(I-P_{\omega}\right) v \\
& -2\left[\alpha(R) r^{2}+\beta(R)+\frac{\gamma(R)}{r}+\frac{\delta(R)}{r^{3}}\right] P_{\omega} v,
\end{aligned}
$$

while

$$
\begin{aligned}
& \Phi_{1, R}[v](x)=v \text { for } x \in B_{0,1}, \\
& \Phi_{1, R}[v](x)=0 \text { for } x \in B_{0, R}^{c} .
\end{aligned}
$$


In the formulas above

$$
\alpha(R)=-\frac{3}{8 R^{3}}+O\left(1 / R^{4}\right), \quad \beta(R)=\frac{9}{8 R}+O\left(1 / R^{2}\right)
$$

while

$$
\gamma(R)=-\frac{3}{4}+O(1 / R), \quad \delta(R)=\frac{1}{4}+O(1 / R)
$$

as $R \rightarrow+\infty$.

If one replaces the boundary condition at $r=R$ with the condition at infinity

$$
\lim _{|x| \rightarrow \infty} \Phi=0
$$

the solution is

$$
\Phi_{1, \infty}[v](x)=\frac{1}{4}\left(\frac{3}{r}+\frac{1}{r^{3}}\right)\left(I-P_{\omega}\right) v+\frac{1}{2}\left(\frac{3}{r}-\frac{1}{r^{3}}\right) P_{\omega} v .
$$

We denote by $\Pi_{1, \infty}[v]$ the associated pressure.

The following relations hold between the pressure fields $\Pi_{1, R}[v]$ and $\Pi_{1, \infty}[v]:$

$$
\begin{aligned}
\omega \cdot \nabla \Phi_{1, R}[v](x)-\Pi_{1, R}[v](x) \omega & =\omega \cdot \nabla \Phi_{1, \infty}[v](x)-\Pi_{1, \infty}(x) \omega \\
& -8 \alpha(R) r\left(I-3 P_{\omega}\right) v+\frac{1}{r^{2}} O\left(\frac{1}{R}\right)
\end{aligned}
$$

in the limit as $R \rightarrow+\infty$. Finally,

$$
\left(\omega \cdot \nabla \Phi_{1, \infty}[v](x)-\Pi_{1, \infty}(x) \omega\right)=-\frac{3}{4}\left(I+3 P_{\omega}\right) \frac{v}{r^{2}}-\frac{3}{4}\left(I-3 P_{\omega}\right) \frac{v}{r^{4}} .
$$

Using the obvious scaling relation

$$
\phi_{\varepsilon}[v](x)=\Phi_{1, r_{\varepsilon} / \varepsilon}[v](x / \varepsilon),
$$

we see that (30) and (31) become, for $r \in\left[\varepsilon, r_{\varepsilon}\right]$

$$
\begin{aligned}
\phi_{\varepsilon}[v](x) & =-\left[4 \alpha_{1}(\varepsilon) r^{2}+2 \beta_{1}(\varepsilon)+\frac{\gamma_{1}(\varepsilon)}{r}-\frac{\delta_{1}(\varepsilon)}{r^{3}}\right]\left(I-P_{\omega}\right) v \\
& -2\left[\alpha_{1}(\varepsilon) r^{2}+\beta_{1}(\varepsilon)+\frac{\gamma_{1}(\varepsilon)}{r}+\frac{\delta_{1}(\varepsilon)}{r^{3}}\right] P_{\omega} v \\
& =A(r)\left(I-P_{\omega}\right) v+B(r) P_{\omega} v
\end{aligned}
$$

with

$$
\alpha_{1}(\varepsilon)=-\frac{3}{8}+O\left(\varepsilon^{2 / 3}\right), \quad \beta_{1}(\varepsilon)=\frac{9}{8} \varepsilon^{2 / 3}+O\left(\varepsilon^{4 / 3}\right),
$$

while

$$
\gamma_{1}(\varepsilon)=-\frac{3}{4} \varepsilon+O\left(\varepsilon^{5 / 3}\right), \quad \delta_{1}(\varepsilon)=\frac{1}{4} \varepsilon^{3}+O\left(\varepsilon^{11 / 3}\right)
$$


Furthermore, for $r \in\left[\varepsilon, r_{\varepsilon}\right]$

$$
\begin{aligned}
\nabla \phi_{\varepsilon}[v] & =-(a(r)+b(r)) \omega \otimes\left(I-P_{\omega}\right) v \\
& +b(r)\left[\left(I-P_{\omega}\right) v \otimes \omega+v \cdot \omega(I-3 \omega \otimes \omega)\right]
\end{aligned}
$$

with

$$
\begin{aligned}
& a(r)=6\left(\alpha_{1} r+\frac{\delta_{1}}{r^{4}}\right), \\
& b(r)=2 \alpha_{1} r-\frac{\gamma_{1}}{r^{2}}-3 \frac{\delta_{1}}{r^{4}} .
\end{aligned}
$$

We also record the following formulas for scalar products :

$$
\begin{array}{r}
\nabla \phi_{\varepsilon}\left[v_{k}\right]: \nabla \phi_{\varepsilon}\left[w\left(x_{k}\right)\right]=\left[(a+b)^{2}+b^{2}\right]\left(v_{k} \cdot w\left(x_{k}\right)-P_{\omega} v_{k} \cdot P_{\omega} w\left(x_{k}\right)\right) \\
+6 b^{2} P_{\omega} v_{k} \cdot P_{\omega} w\left(x_{k}\right)
\end{array}
$$

and

$$
\begin{aligned}
\nabla \phi_{\varepsilon}\left[v_{k}\right]: \nabla w\left(\cdot+x_{k}\right) & =-(a+b) \omega \cdot \nabla\left(v_{k} \cdot w\right)+b \omega \cdot\left(v_{k} \cdot \nabla w\right) \\
& +v_{k} \cdot \omega(a-3 b) \omega \cdot(\omega \cdot \nabla w)+b v_{k} \cdot \omega \nabla \cdot w .
\end{aligned}
$$

In the last formula, we have kept the term $\nabla \cdot w$, although all the vector fields $w$ considered in this work are solenoidal.

\section{PAssing to the Limit}

First, we prove that the correctors defined in (29) converge weakly to 0 in $H_{0}^{1}(\Omega)$ in the vanishing $\varepsilon$ limit.

5.1. Weak convergence of $\mathcal{A}_{\varepsilon}$. Observe that

$\int_{\varepsilon \leq|z| \leq r_{\varepsilon}} G(|z|) P_{z /|z|} v_{k} \cdot P_{z /|z|} w\left(x_{k}\right) d z=\frac{4 \pi}{3} v_{k} \cdot w\left(x_{k}\right) \int_{\varepsilon \leq r \leq r_{\varepsilon}} G(r) r^{2} d r$

for each function $G$ for which the integral on the right-hand side makes sense. Therefore, using (36) and (44), we obtain

$$
\left\|\phi_{\varepsilon}[v]\right\|_{L^{2}(\Omega)}^{2}=\frac{4 \pi}{3}|v|^{2}\left(\int_{\varepsilon}^{r_{\varepsilon}} r^{2}\left(2 A^{2}+B^{2}\right) d r+\varepsilon^{3}\right)
$$

where the last term comes from the integral on $B_{0, \varepsilon}$. 
Since

$$
\begin{aligned}
\int_{\varepsilon}^{r_{\varepsilon}} r^{2}\left(2 A^{2}+B^{2}\right) d r & \leq C\left(\left(\alpha_{1}\right)^{2}\left(r_{\varepsilon}^{7}-\varepsilon^{7}\right)+\left|\alpha_{1} \beta_{1}\right|\left(r_{\varepsilon}^{5}-\varepsilon^{5}\right)\right. \\
& +\left|\alpha_{1} \gamma_{1}\right|\left(r_{\varepsilon}^{4}-\varepsilon^{4}\right)+\left(\beta_{1}\right)^{2}\left(r_{\varepsilon}^{3}-\varepsilon^{3}\right) \\
& +\left(\left|\beta_{1} \gamma_{1}\right|+\left|\alpha_{1} \delta_{1}\right|\right)\left(r_{\varepsilon}^{2}-\varepsilon^{2}\right) \\
& +\left|\beta_{1} \gamma_{1}\right| \log \left(r_{\varepsilon} / \varepsilon\right)+\left(\gamma_{1}\right)^{2}\left(r_{\varepsilon}-\varepsilon\right) \\
& \left.+\left|\delta_{1} \gamma_{1}\right|\left(\frac{1}{\varepsilon}-\frac{1}{r_{\varepsilon}}\right)+\left(\delta_{1}\right)^{2}\left(\frac{1}{\varepsilon^{3}}-\frac{1}{r_{\varepsilon}^{3}}\right)\right)
\end{aligned}
$$

we obtain from (37)-(38) :

(46)

$$
\begin{aligned}
& \left\|\sum_{k=1}^{N} \phi_{\varepsilon}\left[v_{k}\right]\right\|_{L^{2}(\Omega)}^{2}=\sum_{k=1}^{N}\left\|\phi_{\varepsilon}\left[v_{k}\right]\right\|_{L^{2}(\Omega)}^{2}= \\
& \frac{4 \pi}{3} \frac{1}{N} \sum_{k=1}^{N}\left|v_{k}\right|^{2}\left(O\left(N \varepsilon^{3}\right)+O\left(N \varepsilon^{7 / 3}\right)+O\left(N \varepsilon^{9 / 3}\right)+O\left(N \varepsilon^{11 / 3}|\log \varepsilon|\right)\right) \\
& \rightarrow 0,
\end{aligned}
$$

so that $\mathcal{A}_{\varepsilon} \rightarrow 0$ in $L^{2}(\Omega)$.

Next, we consider $(42)$ with $w\left(x_{k}\right)=v_{k}$. Since

$$
\nabla \phi_{\varepsilon}\left[v_{k}\right]: \nabla \phi_{\varepsilon}\left[v_{k}\right]=\left[(a+b)^{2}+b^{2}\right]\left|v_{k}\right|^{2}+\left[(a+b)^{2}+5 b^{2}\right]\left(P_{\omega} v_{k}\right)^{2}
$$

we obtain

$$
\begin{aligned}
& \left\|\nabla \phi_{\varepsilon}\left[v_{k}\right]\right\|_{L^{2}\left(B_{0, r_{\varepsilon}} \backslash B_{0, \varepsilon}\right)}^{2}=\frac{16 \pi}{3}\left|v_{k}\right|^{2} \int_{\varepsilon}^{r_{\varepsilon}}\left[(a+b)^{2}+2 b^{2}\right] r^{2} d r \\
& =\frac{16 \pi}{3}\left|v_{k}\right|^{2}\left(\frac{72}{5} \alpha_{1}^{2}\left(r_{\varepsilon}^{5}-\varepsilon^{5}\right)-3 \gamma_{1}^{2}\left(r_{\varepsilon}^{-1}-\varepsilon^{-1}\right)-\frac{27}{5} \delta_{1}^{2}\left(r_{\varepsilon}^{-5}-\varepsilon^{-5}\right)\right. \\
& \left.-12 \alpha_{1} \gamma_{1}\left(r_{\varepsilon}^{2}-\varepsilon^{2}\right)+24 \alpha_{1} \delta_{1} \ln \frac{r_{\varepsilon}}{\varepsilon}-2 \gamma_{1} \delta_{1}\left(r_{\varepsilon}^{-3}-\varepsilon^{-3}\right)\right)
\end{aligned}
$$

which, together with (37)-(38), gives

$$
\left\|\nabla \phi_{\varepsilon}\left[v_{k}\right]\right\|_{L^{2}\left(B_{0, r_{\varepsilon}} \backslash B_{0, \varepsilon}\right)}^{2} \leq C \varepsilon\left|v_{k}\right|^{2} .
$$

Hence, setting $r_{\varepsilon}=\varepsilon^{1 / 3}$,

$$
\begin{aligned}
\left\|\nabla \mathcal{A}_{\varepsilon}\right\|_{L^{2}(\Omega)}^{2} & \leq C \varepsilon \sum_{k=1}^{N}\left|v_{k}\right|^{2} \\
& =C\left(\int_{\Omega} \int_{v \in \mathbb{R}^{3}} F_{N}(x, v)|v|^{2} d v\right) \leq C^{\prime} .
\end{aligned}
$$


Therefore, there exists a subsequence such that $\mathcal{A}_{\varepsilon} \rightarrow \mathcal{A}$ in $H_{0}^{1}(\Omega)$. Since $\mathcal{A}_{\varepsilon} \rightarrow 0$ in $L^{2}(\Omega), \mathcal{A}=0$ and the whole sequence $\mathcal{A}_{\varepsilon} \rightarrow 0$ in $H_{0}^{1}(\Omega)$.

5.2. Weak convergence of $\mathcal{B}_{\varepsilon}$. Next we prove that the sequence of correctors given by definition (28) converges weakly to 0 in $H_{0}^{1}(\Omega)$.

First we estimate $\left\|\mathcal{B}_{\varepsilon}[w]\right\|_{L^{2}(\Omega)}$ and $\left\|\nabla \mathcal{B}_{\varepsilon}[w]\right\|_{L^{2}(\Omega)}$. In order to do so, we consider the solution $\Psi_{s, r}[\phi]$ of Stokes problem (27) with $w=\phi$.

Using $\Psi_{\varepsilon, 2 \varepsilon}-\Psi_{\varepsilon, \varepsilon^{1 / 3}}$ as test function in problem (27) with $s=\varepsilon$, $r=\varepsilon^{1 / 3}$, we see that

$$
\left\|\nabla \Psi_{\varepsilon, \varepsilon^{1 / 3}}\right\|_{L^{2}\left(B_{0, r_{\varepsilon}} \backslash B_{0, \varepsilon}\right)} \leq\left\|\nabla \Psi_{\varepsilon, 2 \varepsilon}\right\|_{L^{2}\left(B_{0,2} \backslash B_{0, \varepsilon}\right)} .
$$

According to [1], Lemma 2.2.5, formula 2.2.37, p.240, the following holds for each $\eta \in] 0,1\left[\right.$ and $\left.u \in H^{1}\left(B_{0,1}\right)\right)$

$$
\left\|\nabla \Psi_{\eta, 1}[u]\right\|_{L^{2}\left(B_{0,1} \backslash B_{0, \eta}\right)} \leq C\left(\|\nabla u\|_{L^{2}\left(B_{0,1}\right)}+\eta^{1 / 2}\|u\|_{L^{2}\left(B_{0,1}\right)}\right),
$$

where the constant $C$ is uniform in $\eta$ and $u$. Observe that $u(x)=$ $\phi(2 \varepsilon x)$ satisfies

$$
\begin{gathered}
\|u\|_{L^{2}\left(B_{0,1}\right)}=(2 \varepsilon)^{-3 / 2}\|\phi\|_{L^{2}\left(B_{0,2 \varepsilon}\right)}, \\
\|\nabla u\|_{L^{2}\left(B_{0,1}\right)}=(2 \varepsilon)^{-1 / 2}\|\nabla \phi\|_{L^{2}\left(B_{0,2 \varepsilon}\right)},
\end{gathered}
$$

and

$$
\left\|\nabla \Psi_{\varepsilon, 2 \varepsilon}[\phi]\right\|_{L^{2}\left(B_{0,1 \varepsilon} \backslash B_{0, \varepsilon}\right)}=(2 \varepsilon)^{1 / 2}\left\|\nabla \Psi_{\eta, 1}[u]\right\|_{L^{2}\left(B_{0,1} \backslash B_{0,1 / 2}\right)} .
$$

Using successively (53), (49), (50) and (51), (52), we see that

$$
\begin{gathered}
\left\|\nabla \Psi_{\varepsilon, \varepsilon^{1 / 3}}[\phi]\right\|_{L^{2}\left(B_{0, \varepsilon^{1 / 3}} \backslash B_{0, \varepsilon}\right)} \leq(2 \varepsilon)^{1 / 2}\left\|\nabla \Psi_{\eta, 1}[u]\right\|_{L^{2}\left(B_{0,1} \backslash B_{0,1 / 2}\right)} \\
\leq C\left((2 \varepsilon)^{1 / 2}\|\nabla u\|_{L^{2}\left(B_{0,1}\right)}+(1 / 2)^{1 / 2}(2 \varepsilon)^{1 / 2}\|u\|_{L^{2}\left(B_{0,1}\right)}\right) \\
=C\left(\|\nabla \phi\|_{L^{2}\left(B_{0,2 \varepsilon}\right)}+(1 / 2)^{1 / 2}(2 \varepsilon)^{-1}\|\phi\|_{L^{2}\left(B_{0,2 \varepsilon}\right)}\right) .
\end{gathered}
$$

Assuming that $\phi$ is smooth and $\phi(0)=0$ implies that

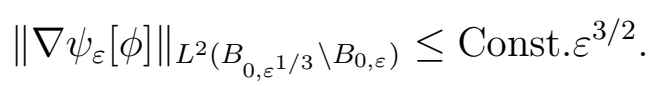

Since

$$
\left\|\nabla \mathcal{B}_{\varepsilon}[w]\right\|_{L^{2}(\Omega)} \leq \sum_{k=1}^{N}\left\|\nabla \phi_{\varepsilon}\left[w\left(x_{k}\right)\right]\right\|_{L^{2}}+\sum_{k=1}^{N}\left\|\nabla \psi_{\varepsilon}\left[w\left(\cdot+x_{k}\right)-w\left(x_{k}\right)\right]\right\|_{L^{2}},
$$


it follows from (48) with $r_{\varepsilon}=\varepsilon^{\frac{1}{3}}$ and $v_{k}=w\left(x_{k}\right)$ and (55) that

$$
\left\|\nabla \mathcal{B}_{\varepsilon}[w]\right\|_{L^{2}(\Omega)}<\text { Const. }
$$

Hence there is a subsequence s.t. $\mathcal{B}_{\varepsilon} \rightarrow \mathcal{B}$ in $H_{0}^{1}(\Omega)$.

On the other hand

$$
\begin{array}{r}
\sum_{k=1}^{N} \psi_{\varepsilon}\left[w\left(\cdot+x_{k}\right)\right]\left(x-x_{k}\right)=\sum_{k=1}^{N} \phi_{\varepsilon}\left[w\left(x_{k}\right)\right]\left(x-x_{k}\right) \\
+\sum_{k=1}^{N} \psi_{\varepsilon}\left[w\left(\cdot+x_{k}\right)-w\left(x_{k}\right)\right]\left(x-x_{k}\right) .
\end{array}
$$

By Poincaré's inequality and (55),

$$
\begin{array}{r}
\sum_{k=1}^{N}\left\|\psi_{\varepsilon}\left[w\left(\cdot+x_{k}\right)-w\left(x_{k}\right)\right]\left(x-x_{k}\right)\right\|_{L^{2}}^{2} \\
\leq \varepsilon^{2 / 3} \sum_{k=1}^{N}\left\|\nabla \psi_{\varepsilon}\left[w\left(\cdot+x_{k}\right)-w\left(x_{k}\right)\right]\left(x-x_{k}\right)\right\|_{L^{2}}^{2} \\
\leq C s t N \varepsilon^{11 / 3} .
\end{array}
$$

Using (46) with $v_{k}=w\left(x_{k}\right)$ shows that

$$
\mathcal{B}_{\varepsilon}=\sum_{k=1}^{N} \psi_{\varepsilon}\left[w\left(\cdot+x_{k}\right)\right] \rightarrow 0
$$

in $\left(L^{2}(\Omega)\right)^{3}$, so that $\mathcal{B}=0$ and the whole sequence

$$
\mathcal{B}_{\varepsilon} \rightarrow 0 \text { in }\left(H_{0}^{1}(\Omega)\right)^{3} \text {. }
$$

Finally, notice that

$$
\left\|\sum_{k=1}^{N} \psi_{\varepsilon}\left[w\left(\cdot+x_{k}\right)-w\left(x_{k}\right)\right]\right\|_{\left(H_{0}^{1}(\Omega)\right)^{3}} \rightarrow 0 .
$$

5.3. Limit of $\int_{\Omega} \nabla \mathcal{A}_{\varepsilon}: \nabla W_{\varepsilon}$. We have

$$
\begin{array}{r}
\int_{\Omega} \nabla \mathcal{A}_{\varepsilon}: \nabla W_{\varepsilon} d x=\int_{\Omega} \nabla \mathcal{A}_{\varepsilon}: \nabla w d x-\int_{\Omega} \nabla \mathcal{A}_{\varepsilon}: \nabla \mathcal{B}_{\varepsilon}[w] d x \\
=\int_{\Omega} \nabla \mathcal{A}_{\varepsilon}: \nabla w d x-\int_{\Omega} \nabla \mathcal{A}_{\varepsilon}: \nabla\left(\sum_{k=1}^{N} \psi_{\varepsilon}\left[w\left(\cdot+x_{k}\right)-w\left(x_{k}\right)\right]\right) d x \\
-\sum_{k=1}^{N} \int_{\varepsilon \leq|z| \leq r_{\varepsilon}} \nabla \phi_{\varepsilon}\left[v_{k}\right]: \nabla \psi_{\varepsilon}\left[w\left(x_{k}\right)\right] d z
\end{array}
$$


In view of (20), we see that

$$
\lim _{\varepsilon \rightarrow 0} \int_{\Omega} \nabla \mathcal{A}_{\varepsilon}: \nabla w d x=0
$$

Moreover, since

$$
\nabla\left(\sum_{k=1}^{N} \psi_{\varepsilon}\left[w\left(\cdot+x_{k}\right)-w\left(x_{k}\right)\right]\right) \rightarrow 0 \text { in }\left(L^{2}(\Omega)\right)^{9}
$$

we also have

$$
\int_{\Omega} \nabla \mathcal{A}_{\varepsilon}: \nabla\left(\sum_{k=1}^{N} \psi_{\varepsilon}\left[w\left(\cdot+x_{k}\right)-w\left(x_{k}\right)\right]\right) d x \rightarrow 0 .
$$

Next we estimate (56). Recalling (44), we have

$$
\int_{\varepsilon \leq|z| \leq r_{\varepsilon}} \nabla \phi_{\varepsilon}\left[v_{k}\right]: \nabla \phi_{\varepsilon}\left[w\left(x_{k}\right)\right] d x=4 \pi v_{k} \cdot w\left(x_{k}\right)\left(\int_{\varepsilon \leq r \leq r_{\varepsilon}} \mathcal{F}(r) r^{2} d r\right),
$$

where (according to (42))

$$
\mathcal{F}(r)=\frac{2}{3}\left[(a+b)^{2}+b^{2}\right]+2 b^{2} .
$$

Therefore,

$$
\begin{array}{r}
\int_{\varepsilon}^{r_{\varepsilon}} r^{2} \mathcal{F}(r) d r=\frac{32}{3} \alpha_{1}^{2}\left(r_{\varepsilon}^{5}-\varepsilon^{5}\right)-6 \delta_{1}^{2}\left(r_{\varepsilon}^{-5}-\varepsilon^{-5}\right)-\frac{10}{3} \gamma_{1}^{2}\left(\frac{1}{r_{\varepsilon}}-\frac{1}{\varepsilon}\right) \\
-\frac{32}{3} \alpha_{1} \gamma_{1}\left(r_{\varepsilon}^{2}-\varepsilon^{2}\right)-4 \gamma_{1} \delta_{1}\left(r_{\varepsilon}^{-3}-\varepsilon^{-3}\right) \\
=\frac{3}{2} \varepsilon+O\left(\varepsilon^{5 / 3}\right) .
\end{array}
$$

Finally,

$$
\int_{\varepsilon \leq|z| \leq r_{\varepsilon}} \nabla \phi_{\varepsilon}\left[v_{k}\right]: \nabla \phi_{\varepsilon}\left[w\left(x_{k}\right)\right] d z=6 \pi \varepsilon v_{k} \cdot w\left(x_{k}\right)+O\left(\varepsilon^{5 / 3}\right),
$$

and

$$
\begin{gathered}
\lim _{\varepsilon \rightarrow 0} \sum_{k=1}^{N} \int_{\varepsilon \leq|z| \leq r_{\varepsilon}} \nabla \phi_{\varepsilon}\left[v_{k}\right]: \nabla \phi_{\varepsilon}\left[w\left(x_{k}\right)\right] d x \\
=\lim _{\varepsilon \rightarrow 0} \sum_{k=1}^{N}\left(6 \pi \varepsilon v_{k} \cdot w\left(x_{k}\right)+O\left(\varepsilon^{5 / 3}\right)\right) \\
=\lim _{\varepsilon \rightarrow 0}\left(6 \pi+O\left(\varepsilon^{2 / 3}\right)\right)(\varepsilon N) \int_{\Omega} \int_{\mathbb{R}^{3}} v \cdot w(x) F_{N}(x, v) d v d x \\
=6 \pi \int_{\Omega} j(x) \cdot w(x) d x .
\end{gathered}
$$


Therefore, we conclude that

$$
\lim _{\varepsilon \rightarrow 0} \int_{\Omega} \nabla \mathcal{A}_{\varepsilon}: \nabla W_{\varepsilon} d x=6 \pi \int_{\Omega} j(x) \cdot w(x) d x .
$$

5.4. Limit for $\int_{\Omega} \nabla U_{\varepsilon}: \nabla \mathcal{B}_{\varepsilon}$. We have

$$
\begin{aligned}
\int_{\Omega} \nabla U_{\varepsilon}: \nabla \mathcal{B}_{\varepsilon}[w] d x & =\int_{\Omega} \nabla U_{\varepsilon}: \nabla\left(\sum_{k=1}^{N} \psi_{\varepsilon}\left[w\left(\cdot+x_{k}\right)-w\left(x_{k}\right)\right]\right) d x \\
& +\sum_{k=1}^{N} \int_{\varepsilon \leq|z| \leq r_{\varepsilon}} \nabla U_{\varepsilon}\left(\cdot+x_{k}\right): \nabla \phi_{\varepsilon}\left[w\left(x_{k}\right)\right] d z
\end{aligned}
$$

Since

$$
\nabla\left(\sum_{k=1}^{N} \psi_{\varepsilon}\left[w\left(\cdot+x_{k}\right)-w\left(x_{k}\right)\right]\right) \rightarrow 0
$$

in $\left(L^{2}(\Omega)\right)^{9}$, we see that

$$
\int_{\Omega} \nabla U_{\varepsilon}: \nabla\left(\sum_{k=1}^{N} \psi_{\varepsilon}\left[w\left(\cdot+x_{k}\right)-w\left(x_{k}\right)\right]\right) d x \rightarrow 0 .
$$

In order to estimate (61), we first integrate by parts, denoting by $n$ the outward unit normal vector to the sphere $\partial B_{x_{k}, r_{\varepsilon}}$ :

$$
\begin{aligned}
I_{k} & =\int_{\varepsilon \leq|z| \leq r_{\varepsilon}} \nabla U_{\varepsilon}\left(\cdot+x_{k}\right): \nabla \phi_{\varepsilon}\left[w\left(x_{k}\right)\right] d z \\
& =-\int_{\varepsilon \leq|z| \leq r_{\varepsilon}} U_{\varepsilon}\left(\cdot+x_{k}\right) \cdot \triangle \phi_{\varepsilon}\left[w\left(x_{k}\right)\right] d z \\
& +\int_{\partial B_{x_{k}, r_{\varepsilon}}}\left(n \cdot \nabla \phi_{\varepsilon}\left[w\left(x_{k}\right)\right]\left(\cdot-x_{k}\right)\right) \cdot U_{\varepsilon} d z .
\end{aligned}
$$

Next we use the definition of $\phi_{\varepsilon}$ to compute

$$
I_{k}=\int_{\partial B_{x_{k}, r_{\varepsilon}}}\left(n \cdot \nabla \phi_{\varepsilon}\left[w\left(x_{k}\right)\right]\left(\cdot-x_{k}\right)-\pi_{\varepsilon}\left(\cdot-x_{k}\right) n\right) \cdot U_{\varepsilon} d z .
$$

At this point, we observe that $n=\omega=\frac{x-x_{k}}{\left|x-x_{k}\right|}$ (that is, $\omega$ is "centered" on $x_{k}$ instead on the origin as in section 4$)$. Since $\nabla \phi_{\varepsilon}(x)=$ 
$\frac{1}{\varepsilon} \nabla \Phi_{1, r_{\varepsilon} / \varepsilon}(x / \varepsilon)$ it follows from (34) that

$$
\begin{aligned}
I_{k}=\frac{1}{\varepsilon} \int_{\partial B_{x_{k}, r_{\varepsilon}}} & \left(\omega \cdot \nabla \Phi_{1, r_{\varepsilon} / \varepsilon}\left[w\left(x_{k}\right)\right]\left(\frac{x-x_{k}}{\varepsilon}\right)-\Pi_{1, \frac{r_{\varepsilon}}{\varepsilon}}\left(\frac{x-x_{k}}{\varepsilon} \omega\right) \cdot U_{\varepsilon} d x\right. \\
=\frac{1}{\varepsilon} & \int_{\partial B_{x_{k}, r_{\varepsilon}}}\left(\omega \cdot \nabla \Phi_{1, \infty}\left[w\left(x_{k}\right)\right]\left(\frac{x-x_{k}}{\varepsilon}\right)-\Pi_{1, \infty}\left(\frac{x-x_{k}}{\varepsilon}\right) \omega\right. \\
& \left.+3\left(\frac{\varepsilon}{r_{\varepsilon}}\right)^{2}\left(I-3 P_{\omega}\right) w\left(x_{k}\right)+O\left(\frac{\varepsilon}{r_{\varepsilon}}\right)^{3}\right) \cdot U_{\varepsilon} d x .
\end{aligned}
$$

By (35),

$$
\begin{array}{r}
I_{k}=\frac{\varepsilon}{r_{\varepsilon}^{2}} \int_{\partial B_{x_{k}}, r_{\varepsilon}}\left(-\frac{3}{4}\left(I+3 P_{\omega}\right) w\left(x_{k}\right)+3\left(I-3 P_{\omega}\right) w\left(x_{k}\right)\right. \\
\left.-\frac{3}{4}\left(I-3 P_{\omega}\right) w\left(x_{k}\right) \frac{\varepsilon^{2}}{r_{\varepsilon}^{2}}\right) \cdot U_{\varepsilon} d x+O\left(\frac{\varepsilon^{2}}{r_{\varepsilon}^{3}}\right),
\end{array}
$$

so that

$$
I_{k}=\frac{\varepsilon}{r_{\varepsilon}^{2}} \int_{\partial B_{x_{k}, r_{\varepsilon}}}\left(-\frac{3}{4}\left(I+3 P_{\omega}\right) w\left(x_{k}\right)+3\left(I-3 P_{\omega}\right) w\left(x_{k}\right)\right) \cdot U_{\varepsilon} d x+O\left(\frac{\varepsilon^{2}}{r_{\varepsilon}^{3}}\right) .
$$

Notice that the same result is obtained in $([1]))$ by a somewhat different procedure.

At this point, we claim the following strong limits in $\left(H^{-1}\left(\mathbb{R}^{3}\right)\right)^{3}$ that hold for any $G \in\left(C_{b}\left(\mathbb{R}^{3}\right)\right)^{3}$ — for a proof, see sec. (6.1)) in the appendix below:

$$
\begin{array}{r}
\sum_{i=1}^{N} r_{\varepsilon} G\left(x_{k}\right) \delta_{\partial B_{x_{k}, r_{\varepsilon}}} \rightarrow 4 \pi \rho(x) G(x) \quad \text { in }\left(H^{-1}\left(\mathbb{R}^{3}\right)\right)^{3}, \\
\sum_{i=1}^{N} r_{\varepsilon} G\left(x_{k}\right) \cdot \omega \omega \delta_{\partial B_{x_{k}, r_{\varepsilon}}} \rightarrow \frac{4 \pi}{3} \rho(x) G(x) \quad \text { in }\left(H^{-1}\left(\mathbb{R}^{3}\right)\right)^{3} .
\end{array}
$$

Since $U_{\varepsilon} \rightarrow U$ converges weakly in $\left(H^{1}(\Omega)\right)^{3}$, we get

$$
\sum_{k=1}^{N} \int_{\varepsilon \leq|z| \leq r_{\varepsilon}} \nabla U_{\varepsilon}: \nabla \psi_{\varepsilon}\left[w\left(\cdot+x_{k}\right)\right] d z \rightarrow-6 \pi \int_{\Omega} w(x) \cdot U \rho(x) d x,
$$

so that

$$
\lim _{\varepsilon \rightarrow 0} \int_{\Omega} \nabla U_{\varepsilon}: \nabla \mathcal{B}_{\varepsilon} d x=-6 \pi \int_{\Omega} w(x) \cdot U \rho(x) d x .
$$

5.5. The limit equation. We start from the weak formulations established in section 3 (that is, equations (17) and (18)). 
5.5.1. The Stokes case. In view of the results established in sections 5.3 and 5.4, we pass to the limit in the Stokes problem (6)-(7)

$$
\int_{\Omega} \nabla U \cdot \nabla w d x=\int_{\Omega} g \cdot w d x+6 \pi\left(\int v \cdot w f d x d v-\int w \cdot U \rho d x\right) .
$$

Therefore, $U$ is the a weak solution of

$$
\left\{\begin{array}{l}
-\triangle U-6 \pi(j-\rho U)+\nabla \Pi=g, \\
\nabla \cdot U=0 \\
\left.U\right|_{\partial \Omega}=0 .
\end{array}\right.
$$

Since the problem above has at most one weak solution, the whole sequence $U_{\varepsilon}$ converges to $U$ in $\left(H^{1}(\Omega)\right)^{3}$.

In addition

$$
\bar{u}_{\varepsilon}=U_{\varepsilon}+\sum_{k=1}^{N} \phi_{\varepsilon}\left[v_{k}\right]\left(x-x_{k}\right) \rightarrow U
$$

in $\left(L^{2}(\Omega)\right)^{3}$, as can be seen from (46).

This finishes the proof of theorem 1, assuming (63) - whose proof is deferred to the appendix below

5.5.2. The Navier-Stokes case. Likewise, for the Navier-Stokes problem (14), (7) in the limit as $\varepsilon \rightarrow 0$

$$
\begin{aligned}
\nu \int_{\Omega} \nabla U \cdot \nabla w d x & =\int_{\Omega} U \otimes U: \nabla w d x \\
& +\int_{\Omega} g \cdot w d x+6 \pi\left(\int v \cdot w f d x d v-\int w \cdot U \rho d x\right) .
\end{aligned}
$$

Given $\rho, j$ and $g$, there exists $\nu_{0}>0$ large enough, so that, for each $\nu>\nu_{0}$, the problem

$$
\left\{\begin{array}{l}
U \cdot \nabla U-\nu \triangle U(x)-6 \pi(j-\rho U)+\nabla \Pi=g, \\
\nabla \cdot U=0, \\
\left.U\right|_{\partial \Omega}=0,
\end{array}\right.
$$

has a unique weak solution $U \in\left(H_{0}^{1}(\Omega)\right)^{3}$.

Hence the whole sequence $U_{\varepsilon}$ converges weakly to $U$ in $\left(H_{0}^{1}(\Omega)\right)^{3}$ as $\varepsilon \rightarrow 0$.

As in the Stokes case, (46) implies that

$$
\bar{u}_{\varepsilon}=U_{\varepsilon}+\sum_{k=1}^{N} \phi_{\varepsilon}\left[v_{k}\right]\left(x-x_{k}\right) \rightarrow U
$$

in $\left(L^{2}(\Omega)\right)^{3}$. This completes the proof of theorem 2 - assuming again that the limits in $(63)$ hold. 


\section{ApPEndix}

6.1. Proof of (63). We closely follow the method described in [7] in the periodic setting. Given $G \in\left(C_{b}\left(\mathbb{R}^{3}\right)\right)^{3}$, we consider two auxiliary problems in $\bigcup_{k=1}^{N} B_{x_{k}, r_{\varepsilon}}$ : for $k=1, \ldots, N$

$$
\left\{\begin{array}{l}
-\triangle \xi_{\varepsilon}=-3 G\left(x_{k}\right), \\
\left.\frac{\partial \xi_{\varepsilon}}{\partial n}\right|_{\partial B_{x_{k}, r_{\varepsilon}}}=r_{\varepsilon} G\left(x_{k}\right)
\end{array}\right.
$$

and

$$
\left\{\begin{array}{l}
-\triangle \chi_{\varepsilon}=-G\left(x_{k}\right)-\frac{r}{r_{\varepsilon}}\left(6\left(G\left(x_{k}\right) \cdot \omega\right) \omega+2 G\left(x_{k}\right)\right)+3 G\left(x_{k}\right), \\
\left.\frac{\partial \chi_{\varepsilon}}{\partial n}\right|_{\partial B_{x_{k}, r_{\varepsilon}}}=r_{\varepsilon}\left(G\left(x_{k}\right) \cdot \omega\right) \omega .
\end{array}\right.
$$

Next we extend $\xi_{\varepsilon}$ and $\chi_{\varepsilon}$ by 0 in the complement of $\bigcup_{k=1}^{N} B_{x_{k}, r_{\varepsilon}}$. Computing the Laplacian of $\xi_{\varepsilon}$ and $\chi_{\varepsilon}$ in the sense of distributions in the whole Euclidean space, we get

$$
\begin{aligned}
-\triangle \xi_{\varepsilon} & =-3 \sum_{k=1}^{N} G\left(x_{k}\right) 1_{B_{x_{k}, r_{\varepsilon}}}+\sum_{k=1}^{N} r_{\varepsilon} G\left(x_{k}\right) \delta_{\partial B_{x_{k}, r_{\varepsilon}}} \\
& =-3 N 1_{B_{0, r_{\varepsilon}}} *\left(G \rho_{N}\right)+\sum_{k=1}^{N} r_{\varepsilon} G\left(x_{k}\right) \delta_{\partial B_{x_{k}, r_{\varepsilon}}}
\end{aligned}
$$

$$
\begin{aligned}
-\triangle \chi_{\varepsilon} & =-\sum_{k=1}^{N} G\left(x_{k}\right) 1_{B_{x_{k}, r_{\varepsilon}}} \\
& +\sum_{k=1}^{N}\left\{\frac{r}{r_{\varepsilon}}\left(6\left(G\left(x_{k}\right) \cdot \omega\right) \omega+2 G\left(x_{k}\right)\right)-3 G\left(x_{k}\right)\right\} 1_{B_{x_{k}}, r_{\varepsilon}} \\
& +\sum_{k=1}^{N} r_{\varepsilon}\left(G\left(x_{k}\right) \cdot \omega\right) \omega \delta_{\partial B_{x_{k}, r_{\varepsilon}}} \\
& =-N 1_{B_{0, r_{\varepsilon}}} *\left(G \rho_{N}\right)+\sum_{k=1}^{N} r_{\varepsilon}\left(G\left(x_{k}\right) \cdot \omega\right) \omega \delta_{\partial B_{x_{k}, r_{\varepsilon}}} \\
& +\sum_{k=1}^{N}\left\{\frac{r}{r_{\varepsilon}}\left(6\left(G\left(x_{k}\right) \cdot \omega\right) \omega+2 G\left(x_{k}\right)\right)-3 G\left(x_{k}\right)\right\} 1_{B_{x_{k}, r_{\varepsilon}}} .
\end{aligned}
$$


The solutions of the two auxiliary problems above are

$$
\begin{aligned}
\xi_{\varepsilon}(x) & =\sum_{i=1}^{N} \frac{\left|x-x_{k}\right|^{2}-r_{\varepsilon}^{2}}{2} G\left(x_{k}\right) 1_{B_{x_{k}, r_{\varepsilon}}}, \\
\chi_{\varepsilon}(x) & =\sum_{i=1}^{N}\left(\frac{\left|x-x_{k}\right|^{3}}{r_{\varepsilon}}-\left|x-x_{k}\right|^{2}\right)\left(G\left(x_{k}\right) \cdot \omega\right) \omega 1_{B_{x_{k}, r_{\varepsilon}}},
\end{aligned}
$$

while their gradients are given by

$$
\begin{aligned}
\nabla \xi_{\varepsilon}(x) & =\sum_{k=1}^{N}\left|x-x_{k}\right|\left(\omega \otimes G\left(x_{k}\right)\right) 1_{B_{x_{k}, r_{\varepsilon}}}, \\
\nabla \chi_{\varepsilon}(x) & =\sum_{k=1}^{N} \frac{\left|x-x_{k}\right|^{2}}{r_{\varepsilon}}\left(\left(\omega \otimes G\left(x_{k}\right)\right) \cdot \omega\right) \omega 1_{B_{x_{k}}, r_{\varepsilon}} \\
& +\sum_{k=1}^{N}\left(\frac{\left|x-x_{k}\right|^{2}}{r_{\varepsilon}}-\left|x-x_{k}\right|\right) \\
& \times\left(\left(G\left(x_{k}\right) \cdot \omega\right) I+G\left(x_{k}\right) \otimes \omega\right) 1_{B_{x_{k}, r_{\varepsilon}}}
\end{aligned}
$$

with $\omega=\frac{x-x_{k}}{\left|x-x_{k}\right|}$.

Then, we estimate

$$
\begin{aligned}
\left\|\xi_{\varepsilon}\right\|_{L^{2}\left(\mathbb{R}^{3}\right)}^{2} & \leq \text { Const. } r_{\varepsilon}^{4} \int G^{2} \rho_{N} d x=O\left(r_{\varepsilon}^{4}\right), \\
\left\|\nabla \xi_{\varepsilon}\right\|_{L^{2}\left(\mathbb{R}^{3}\right)}^{2} & \leq \text { Const. } r_{\varepsilon}^{2} \int G^{2} \rho_{N} d x=O\left(r_{\varepsilon}^{2}\right), \\
\left\|\chi_{\varepsilon}\right\|_{L^{2}\left(\mathbb{R}^{3}\right)}^{2} & \leq \text { Const., } r_{\varepsilon}^{4} \int G^{2} \rho_{N} d x=O\left(r_{\varepsilon}^{4}\right), \\
\left\|\nabla \chi_{\varepsilon}\right\|_{L^{2}\left(\mathbb{R}^{3}\right)}^{2} & \leq \text { Const.t } r_{\varepsilon}^{2} \int G^{2} \rho_{N} d x=O\left(r_{\varepsilon}^{2}\right) .
\end{aligned}
$$

Therefore, $\xi_{\varepsilon}$ and $\chi_{\varepsilon} \rightarrow 0$ in $\left(H^{1}\right)^{3}$, so that both

$$
\Delta \xi_{\varepsilon} \text { and } \Delta \chi_{\varepsilon} \rightarrow 0 \text { in }\left(H^{-1}\right)^{3} .
$$

Next, we recall that $N 1_{B_{0, r_{\varepsilon}}} \rightarrow \frac{4 \pi}{3} \delta_{0}$ weakly in the sense of measures; hence

$$
N 1_{B_{0, r_{\varepsilon}}} *\left(G \rho_{N}\right) \rightarrow \frac{4 \pi}{3} \rho G
$$

weakly in the sense of measures. Furthermore

$$
\left\|N 1_{B_{x_{k}, r_{\varepsilon}}} *\left(G \rho_{N}\right)\right\|_{L^{\infty}} \leq\|G\|_{L^{\infty}}
$$


so that, by the Rellich compactness theorem, the limit (71) holds in the strong topology of $\left(H_{l o c}^{-1}\right)^{3}$.

Going back to (68), we conclude from (70) that

$$
\sum_{k=1}^{N} r_{\varepsilon} G\left(x_{k}\right) \delta_{\partial B_{x_{k}, r_{\varepsilon}}} \rightarrow 4 \pi \rho G \text { in }\left(H_{l o c}^{-1}\right)^{3}
$$

strongly.

Next, we apply the same procedure to the second term on the right hand side of (69).

First, we observe that the last term on that right hand side is bounded in $L^{\infty}\left(\mathbb{R}^{3}\right)$ by $11\|G\|_{L^{\infty}}$, while

$$
\int_{\Omega}\left(\phi(x) \cdot \sum_{k=1}^{N}\left\{\frac{r}{r_{\varepsilon}}\left(6\left(G\left(x_{k}\right) \cdot \omega\right) \omega+2 G\left(x_{k}\right)\right)-3 G\left(x_{k}\right)\right\} 1_{B_{x_{k}, r_{\varepsilon}}}\right) d x \rightarrow 0
$$

for each $\phi \in\left(\mathcal{D}\left(\mathbb{R}^{3}\right)\right)^{3}$. Applying the Rellich compactness theorem again shows that the convergence (72) holds in the strong topology of $\left(H_{l o c}^{-1}\right)^{3}$.

Going back to (69) and using (70), (71) and (72) shows that

$$
\sum_{k=1}^{N} r_{\varepsilon}\left(G\left(x_{k}\right) \cdot \omega\right) \omega \delta_{\partial B_{x_{k}, r_{\varepsilon}}} \rightarrow \frac{4 \pi}{3} \rho G \text { in }\left(H^{-1}\right)^{3}
$$

strongly.

6.2. Solution of Stokes' problem in an annulus. We first prove the explicit formula for $\Phi_{1, R}$ in (30), (31) and (32), by the same method as in $[12] \S 20$. By symmetry, we seek $\Phi_{1, R}$ in the form $\Phi_{1, R}[v]=$ $\operatorname{curl} \operatorname{curl}(f(r) v)$ (where $r=|x|)$. Then

$$
\left(\frac{\partial^{2}}{\partial r^{2}}+\frac{2}{r} \frac{\partial}{\partial r}\right)\left(\frac{\partial^{2}}{\partial r^{2}}+\frac{2}{r} \frac{\partial}{\partial r}\right) f(r)=\text { Const. }
$$

so that

$$
f^{\prime}(r)=\alpha r^{3}+\beta r+\gamma+\frac{\delta}{r^{2}}
$$


Denoting by $P_{\omega}$ the orthogonal projection on $\omega=x / r$ we arrive at formula (30) :

$$
\begin{aligned}
\Phi_{1, R}[v](x)= & -\left(f^{\prime \prime}+\frac{f^{\prime}}{r}\right)\left(I-P_{\omega}\right) v-2 \frac{f^{\prime}}{r} P_{\omega} v \\
= & -\left[4 \alpha(R) r^{2}+2 \beta(R)-\frac{\delta(R)}{r^{3}}+\frac{\gamma(R)}{r}\right]\left(I-P_{\omega}\right) v \\
& -2\left[\alpha(R) r^{2}+\beta(R)+\frac{\delta(R)}{r^{3}}+\frac{\gamma(R)}{r}\right] P_{\omega} v .
\end{aligned}
$$

Because of the boundary conditions, the constants $\alpha, \beta, \gamma, \delta$ in the formula above satisfy the following system of equations :

$$
\begin{aligned}
3\left(R^{5}-1\right) \alpha & +\left(R^{3}-1\right) \beta=\frac{1}{2}, \\
5\left(R^{3}-1\right) \alpha & +3(R-1) \beta=\frac{3}{2}, \\
\frac{3}{2} \alpha & +\frac{1}{2} \beta+\frac{1}{4}=\delta, \\
-\frac{5}{2} \alpha & -\frac{3}{2} \beta-\frac{3}{4}=\gamma,
\end{aligned}
$$

leading to the estimates (31) and (32) :

$$
\begin{array}{cc}
\alpha=-\frac{3}{8 R^{3}}+O_{R \rightarrow+\infty}\left(1 / R^{4}\right), & \beta=\frac{9}{8 R}+O_{R \rightarrow+\infty}\left(1 / R^{2}\right), \\
\gamma=-\frac{3}{4}+O_{R \rightarrow+\infty}(1 / R), & \delta=\frac{1}{4}+O_{R \rightarrow+\infty}(1 / R) .
\end{array}
$$

Next we compute the pressure for the above flow; for simplicity, we first write down the following table :

$$
\begin{array}{ll}
\nabla r=\omega, & \nabla \omega=\frac{I-\omega \otimes \omega}{r}, \\
\nabla \cdot \omega=\frac{2}{r}, & \omega \cdot \nabla \omega=0, \\
\nabla(\omega \cdot v)=\frac{v-P_{\omega} v}{r}, & \\
\omega \cdot P_{\omega} v=\omega \cdot v, & \nabla \cdot(a \otimes b)=(\nabla \cdot a) b+a \cdot \nabla b,
\end{array}
$$

so that

$$
\begin{aligned}
\nabla P_{\omega} v & =\frac{v-P_{\omega} v}{r} \otimes \omega+(\omega \cdot v) \nabla \omega \text { and } \omega \cdot \nabla P_{\omega} v=0 \\
\nabla \cdot P_{\omega} v & =\frac{2}{r} \omega \cdot v, \quad \Delta \omega=-\frac{2}{r^{2}} \omega \\
\triangle P_{\omega} v & =2\left(\frac{v-3 P_{\omega} v}{r^{2}}\right) .
\end{aligned}
$$


To find the pressure, observe that

$\triangle \Phi_{1, R}[v](x)=-\left[20 \alpha-\frac{5 \alpha}{r^{3}}-\frac{3 \beta}{r^{3}}-\frac{3}{2 r^{3}}\right] v-3\left[\frac{5 \alpha}{r^{3}}+\frac{3 \beta}{r^{3}}+\frac{3}{2 r^{3}}\right] P_{\omega} v$

so that, up to some unessential additive constant,

$$
\Pi_{1, R}=-\left[\left(20 \alpha r-\frac{3}{2 r^{2}}-\frac{5 \alpha+3 \beta}{r^{2}}\right) \omega \cdot P_{\omega} v\right] .
$$

Letting $R \rightarrow+\infty$ in the previous expressions leads to formula (33) (see also [12]) :

$$
\Phi_{1, \infty}[v](x)=\frac{1}{4}\left(\frac{3}{r}+\frac{1}{r^{3}}\right)\left(I-P_{\omega}\right) v+\frac{1}{2}\left(\frac{3}{r}-\frac{1}{r^{3}}\right) P_{\omega} v
$$

and

$$
\Pi_{1, \infty}=\frac{3}{2 r^{2}} \omega \cdot P_{\omega} v
$$

Obviously

$$
\begin{aligned}
\Phi_{1, R}[v](x) & =\Phi_{1, \infty}[v](x)-\left[4 \alpha(R) r^{2}+2 \beta(R)\right]\left(I-P_{\omega}\right) v \\
& -2\left[\alpha(R) r^{2}+\beta(R)\right] P_{\omega} v+\frac{1}{r} O\left(\frac{1}{R}\right), \\
\omega \cdot \nabla \Phi_{1, R}[v](x) & =\omega \cdot \nabla \Phi_{1, \infty}[v](x) \\
& -4 \alpha(R) r\left(2 I-P_{\omega}\right) v+\frac{1}{r^{2}} O\left(\frac{1}{R}\right),
\end{aligned}
$$

while

$$
\Pi_{1, R}=\Pi_{1, \infty}-20 \alpha(R) r \omega \cdot v+\frac{1}{r^{2}} O\left(\frac{1}{R}\right) .
$$

as $R \rightarrow+\infty$.

Formula (34) follows as a consequence:

$$
\begin{aligned}
\omega \cdot \nabla \Phi_{1, R}-\Pi_{1, R} \omega & =\omega \cdot \nabla \Phi_{1, \infty}-\Pi_{1, \infty} \omega \\
& -8 \alpha(R) r\left(I-3 P_{\omega}\right) v+\frac{1}{r^{2}} O\left(\frac{1}{R}\right) .
\end{aligned}
$$

From (73) and (74), we arrive at formula (35) :

$$
\omega \cdot \nabla \Phi_{1, \infty}[v](x)-\Pi_{1, \infty} \omega=-\frac{3}{4}\left(I+3 P_{\omega}\right) \frac{v}{r^{2}}-\frac{3}{4}\left(I-3 P_{\omega}\right) \frac{v}{r^{4}} .
$$

Finally, we derive formulas (42), (43). First rewrite formula (39) for $\nabla \phi_{\varepsilon}[v]$ with $r \in\left[\varepsilon, r_{\varepsilon}\right]$ in the form

$$
\nabla \phi_{\varepsilon}[v]=-a(r) N(v)+b(r)[M(v)+v \cdot \omega(I-3 \omega \otimes \omega)]
$$


where $M$ and $N$ are two matrix-valued, linear functions of $v$ :

$$
\begin{aligned}
M(v) & =\left(I-P_{\omega}\right) v \otimes \omega-\omega \otimes\left(I-P_{\omega}\right) v, \\
N(v) & =\omega \otimes\left(I-P_{\omega}\right) v,
\end{aligned}
$$

while

$$
a(r)=6\left(\alpha_{1} r+\frac{\delta_{1}}{r^{4}}\right), \quad b(r)=2 \alpha_{1} r-\frac{\gamma_{1}}{r^{2}}-3 \frac{\delta_{1}}{r^{4}} .
$$

For each $v, w \in \mathbb{R}^{3}$ and each $\omega \in S^{2}$

$$
\begin{aligned}
\frac{1}{2} M(v): M(w) & =N(v): N(w)=-M(v): N(w)=\left(v \cdot w-P_{\omega} v \cdot P_{\omega} w\right), \\
M(v): \omega \otimes \omega & =M(v): I=N(v): \omega \otimes \omega=N(v): I=0, \\
\omega \otimes \omega: I & =\omega \otimes \omega: \omega \otimes \omega=1, \quad I: I=3 .
\end{aligned}
$$

Now (42) and (43) follow from (76) by elementary manipulations involving the identities recalled above.

\section{REFERENCES}

[1] G. Allaire. Homogenization of the Navier-Stokes equations in open sets perforated with tiny holes. Arch. Rational Mech. Anal., 113, p. 209-259, 1991.

[2] A.A. Amsden, P.J. O'Rourke, T.D. Butler. A computer program for chemically reactive flows with sprays. Report \# LA-11560-MS, Los Alamos National Laboratory, 1989.

[3] G.K. Batchelor. Sedimentation in a dilute suspension of spheres. J. Fluid Mech. 52 (1972), 245-268.

[4] C. Baranger, L. Desvillettes. Coupling Euler and Vlasov equations in the context of sprays: the local-in-time, classical solutions. J. Hyperbolic Differ. Equ. 3 (2006), 1-26.

[5] R.E. Caflisch, J. H.C. Luke. Variance in the sedimentation speed of a suspension. Phys. Fluids 28 (1985), 759-760.

[6] R.E. Caflisch, J. Rubinstein. "Lectures on the mathematical theory of multiphase flows" Courant Institute Lecture Notes, New-York, 1984.

[7] D. Cioranescu, F. Murat. Une terme étrange venu d'ailleurs. In "Nonlinear Partial Differential Equations and their Applications", Collège de France Seminar, Vol. 2, Research Notes in Mathematics, 60, p.98-138, (1982).

[8] F. Feuillebois. Sedimentation in a dispersion with vertical inhomogeneities. J. Fluid Mech. 139 (1984), 145-171.

[9] P.-E. Jabin. Various levels of models for aerosols. Math.Models and Methods in Appl. Sci. 12 (2002), 903-919.

[10] P.-E. Jabin, F. Otto. Identification of the dilute regime in particle sedimentation. Commun. Math. Phys. 250 (2004), 415-432.

[11] O.A. Ladyzhenskaya: "The mathematical theory of viscous incompressible flow". Mathematics and its Applications, Vol. 2 Gordon and Breach, Science Publishers, New York-London-Paris 1969.

[12] L.D. Landau, E.M. Lifshitz. Course of theoretical physics. Vol. 6. Fluid mechanics. Pergamon Press, Oxford, 1987. 
[13] V. A. L'vov, E. Ya. Khruslov. O возмуштении вязкой несжимаемой жидкости мелкими частицами(Russian) [Perturbation of a viscous incompressible fluid by small particles] Theoretical and applied questions of differential equations and algebra (Russian) 267 (1978), 173-177.

[14] P.J. O'Rourke. Collective drop effects on vaporizing liquid sprays. $\mathrm{PhD}$ thesis, Los Alamos National Laboratory, 1981.

[15] J. Rubinstein, J. Keller: Particle distribution functions in suspensions. Phys. Fluids A 1 (1989), 1632-1641.

[16] J. Rubinstein: On the macroscopic description of slow viscous flow past a random array of spheres. J. Statist. Phys. 44 (1986), 849-863.

(L.D.) Ecole Normale Supérieure de Cachan, CMla, 61, Av. Du Pdt. Wilson, F94235 Cachan Cedex

E-mail address: desville@cmla.ens-cachan.fr

(F.G.) Laboratoire J.-L. Lions, Université Pierre-et-Marie Curie, Boîte Courrier 187, 75252 Paris Cedex 05, \& Centre de Mathématiques

Laurent Schwartz, Ecole Polytechnique, F91128 Palaiseau Cedex

E-mail address: golse@math.polytechnique.fr

(V.R.) Dipartimento di Metodi e Modelli Matematici, Universitì di Palermo, Viale delle Scienze Edificio 8, I90128 Palermo

E-mail address: ricci@unipa.it 